\title{
Influence of visfatin's gene variations on late diabetic complications
}

\author{
Corresponding author: \\ Wioletta Szywacz, SPSK No 1 \\ 3-go Maja 13-15 Str. \\ 41-800 Zabrze, Poland \\ e-mail:wiolettaszywacz@gmail.com
}

Medical Research Journal 2021;

Volume 6, Number 1, 28-32

10.5603/MRJ.a2021.0004

Copyright (C) 2021 Via Medica

ISSN 2451-2591

e-ISSN 2451-4101

\begin{abstract}
Visfatin (nicotinamide phosphoribosyltransferase) is an adipokine that performs many functions in the organism. It can be expressed in different tissues such as the brain, kidneys and visceral adipose tissue. Visfatin takes part in many molecular processes including apoptosis, inflammation, cell proliferation. It affects glucose metabolism and is involved in the pathogenesis of diabetes, insulin resistance, atherosclerosis and obesity. Moreover, studies suggest that visfatin also may be associated with the development of diabetic nephropathy and retinopathy.

The goal of the study is the assessment of the influence of different visfatin's gene variants on the occurrence of late diabetic complications.

The study group consisted of 272 patients with diabetes - 139 men and 133 women from Southern Poland. Selected DNA fragments were amplificated and marked. Visfatin's gene in rs4730153 was examined. The Real-Time PCR was conducted with fluorescence-labelled probes.

The most common genotypes were heterozygote AG- 138 patients (51\%) and homozygote GG- 89 patients (33\%)

In the study group, there were 92 diabetics with retinopathy, 26 with nephropathy, 88 with neuropathy and 103 with macroangiopathy.

It has been assessed using the $\chi 2$ test that there are no differences between the variability of different variants of visfatin's gene in the distribution of genotypes. According to Hardy-Weinberg's test, the variety of population is maintained.

Key words: visfatin, diabetes mellitus, SNP, polymorphism, gene, late complications
\end{abstract}

Med Res J 2021; 6 (1): 28-32

\section{Introduction}

Diabetes mellitus is a silent pandemic of the 21 st century [1]. Its prevalence is estimated to rise from 425 million people in 2017 to 629 million by 2045 [2]. Many patients with type 2 diabetes (T2DM) develop microvascular complications (approximately $40 \%$ of those develop diabetic kidney disease (DKD), which is a leading cause of the end-stage renal disease (ESRD) globally [3] and approximately $40-80 \%$ develop diabetic retinopathy (DR) [4]

DKD as well as DR are often diagnosed at advanced stages [5]. The multifactorial pathogenesis of DKD and DR consists of a combination of metabolic, environmental, and genetic factors [1]. A prior genome-wide association study (GWAS) identified genes with polymorphisms associated with an increased incidence of DKD and DR [6]. However, further studies to prove the role of these polymorphisms in DKD and DR occurrence and progression in different diabetic populations are needed, to guide strategies that prevent and treat DKD and DR.

Visfatin, a recently discovered adipocytokine is the extracellular isoform of the NAMPT enzyme. Its gene is located on chromosome 7 and the exact location is as follows: band 7q22.3, starting 106,248,298 bp, ending $106,286,326$ bp. Visfatin can cause an insulin-mimetic effect in cells. As it downregulates the amount of glucose release from the liver, visfatin accelerates triglycerides synthesis and increases glucose metabolism in monocytes and adipocytes. It plays an important role in the pathogenesis of T2DM and its complications such as diabetic nephropathy and retinopathy. Nevertheless, there are limited studies about the association between visfatin and diabetic complications in these diseases.

The role of visfatin in diabetic retinopathy is poorly understood. In humans, only one study investigated the 
differences in visfatin concentrations between patients with and without diabetic retinopathy. Y. Wang et al. have found elevated vitreous and serum level of visfatin in diabetic patients with proliferative diabetic retinopathy compared to these with nonproliferative diabetic retinopathy, without diabetic retinopathy and nondiabetic controls [7].

Patients with diabetic nephropathy show markedly increased serum levels of visfatin comparing to the non-diabetic group [8-10]. Mageswari R et al. suggest that visfatin level could be an index of severity of diabetic kidney disease. Many studies are investigating the role of Single Nucleotide Polymorphism (SNP) in the visfatin gene. It has been researched that the variations are involved in the pathogenesis of diabetes, obesity as well as may regulate plasma insulin levels and plasma glucose levels.

In light of the positive GWAS outcomes in relation to the visfatin gene and the contradictory outcomes of follow-up studies - as well as the lack of studies performed in a Polish population - this study assessed the association of rs 4730153 visfatin gene variants with DKD and DR in a group of Polish T2DM patients (the industrial region of Silesia, Poland).

The presented study is the first one to investigate the influence of SNP in the visfatin gene on diabetic retinopathy and nephropathy occurrence.

\section{Materials and methods}

Materials used for this genetic study were samples of venous blood taken from willing subjects. All patients partaking in this study have signed written consent. The study group consisted of 272 individuals. Among them, 139 were men and 133 women. All of these patients had been previously diagnosed with T2DM.

For the time needed to gather the representative group for research, blood samples collected from subjects were stored at the proper temperature at minus 70 Celsius degrees.

In the laboratory of Clinical Hospital 1 in Zabrze, the DNA material was isolated from obtained blood. The next step was to prepare a proper concentration of the DNA which was $15 \mathrm{ng} / \mu \mathrm{l}$. Then with a spectrophotometer, the purity of samples was checked.

Using fluorescent-labelled TaqMan Pre-designed SNP Genotyping Assay probes allelic discrimination was performed in Roche Lightcycler 96 thermocycler. Alleles were marked as A in VIC and G in FAM.

Finally, statistical analysis was made to present the result of the study. The significance between distributions of genotypes and alleles, presence of diabetic retinopathy, nephropathy, neuropathy and macroangiopathy were tested using Pearson's $\chi 2$ test. Non-parametric ANOVA analysis was used to examine the association of visfatin polymorphism in rs4730153 with the occurrence of late complications of diabetes mellitus. $P$ values $<0.05$ were considered statistically significant. The statistical software STATISTICA 13 for Windows (TIBCO Software Inc., Palo Alto, CA, USA) was used to perform all analyses.

\section{Results}

In order to analyze the dependence between visfatin's gene in rs4720153 and late complications of T2DM, the number of each patients' alleles and genotypes were profiled with clinical data and occurrence of complications.

Among studied patients 29 were in the healthy weight range (10,5\%), 115 overweight $(42,5 \%)$ and 128 obese (47\%) due to the WHO Body Mass Index (BMI) (Tab.1). The waist to hip ratio (WHR) of patients was also assessed with similar results: 17 patients with normal ratio $(6,25 \%), 64$ over-weight $(23,5 \%)$ and 191 obese $(70,25 \%)$.

Genotype distribution of the patients complied with populational- presented heterozygote (AG) genotype occurred most often (51\%) with AA or GG respectively 17 and $33 \%$. The distribution was compatible with Hardy-Weinberg equilibrium (Tab. 2).

Regarding treatment schedule, at the moment of testing 101 individuals were treated with insulin injections, 171 were taking oral medicaments and 72 were prescribed both of them. Hypertension was present in $74.3 \%$ of patients (202 patients). Patients with dyslipidaemia stated $54,8 \%$ (149) of all group.

Table 1. Distribution of genotypes of visfatin's SNP in rs4730153 in reference to BMI

\begin{tabular}{|c|c|c|c|c|}
\hline & & $\begin{array}{l}\text { Normal body } \\
\text { weight }\end{array}$ & Overweight & Obese \\
\hline \multirow[t]{2}{*}{$A A$} & $\mathrm{n}$ & 4 & 26 & 15 \\
\hline & $\%$ & 9 & 58 & 33 \\
\hline \multirow[t]{2}{*}{$A G$} & $\mathrm{n}$ & 15 & 57 & 66 \\
\hline & $\%$ & 11 & 41 & 48 \\
\hline \multirow[t]{2}{*}{ GG } & $\mathrm{n}$ & 10 & 32 & 47 \\
\hline & $\%$ & 11 & 36 & 53 \\
\hline
\end{tabular}

$p=0,1962$

Table 2. Distribution of genotypes of visfatin's SNP in rs4730153 in the research group

\begin{tabular}{lcc}
\hline Genotype & $\begin{array}{c}\text { Amount of } \\
\text { carriers }\end{array}$ & $\begin{array}{c}\text { Percentage of } \\
\text { group }\end{array}$ \\
\hline $\mathrm{AA}$ & 45 & $17 \%$ \\
$\mathrm{AG}$ & 138 & $51 \%$ \\
$\mathrm{GG}$ & 89 & $33 \%$ \\
\hline
\end{tabular}


Table 3. Distribution of genotypes of visfatin's SNP in rs4730153 in reference to retinopathy occurrence

\begin{tabular}{lcccccc}
\hline Genotypes & \multicolumn{2}{c}{ With retinopathy } & \multicolumn{2}{c}{ Without retinopathy } & p-value & OR (95\% Cl) \\
\cline { 2 - 5 } & $\mathbf{N}$ & $\%$ & $\mathbf{N}$ & $\%$ & & \\
\hline $\mathrm{AA}$ & 13 & 28.89 & 32 & 71.11 & - & 1.00 (Reference) \\
$\mathrm{AG}$ & 43 & 31.16 & 95 & 68.84 & 0.7742 & $1.114(0.532-2.332)$ \\
$\mathrm{GG}$ & 36 & 40.45 & 53 & 59.55 & 0.1914 & $1.672(0.773-3.615)$ \\
$\mathrm{AG}+\mathrm{GG}$ & 79 & 34.80 & 148 & 65.20 & 0.4447 & $0.761(0.378-1.533)$ \\
\hline
\end{tabular}

Table 4. Distribution of genotypes of visfatin's SNP in rs4730153 in reference to nephropathy occurrence

\begin{tabular}{lcccccc}
\hline Genotypes & \multicolumn{2}{c}{ With nephropathy } & \multicolumn{2}{c}{ Without nephropathy } & p-value & OR (95\% Cl) \\
\cline { 2 - 5 } & $\mathbf{N}$ & $\%$ & $\mathbf{N}$ & $\%$ & & \\
\hline $\mathrm{AA}$ & 2 & 4.44 & 45 & 95.56 & - & 1.00 (Reference) \\
$\mathrm{AG}$ & 12 & 8.70 & 126 & 91.30 & 0.3606 & $2.048(0.441-9.518)$ \\
$\mathrm{GG}$ & 12 & 13.48 & 77 & 86.52 & 0.1245 & $3.351(0.716-15.673)$ \\
$\mathrm{AG}+\mathrm{GG}$ & 24 & 10.57 & 203 & 89.43 & 0.2165 & $2.542(0.579-11.162)$ \\
\hline
\end{tabular}

Table 5. Distribution of genotypes of visfatin's SNP in rs4730153 in reference to neuropathy occurrence

\begin{tabular}{lcccccc}
\hline Genotypes & \multicolumn{2}{c}{ With neuropathy } & \multicolumn{2}{c}{ Without neuropathy } & p-value & OR (95\% Cl) \\
\cline { 2 - 5 } & $\mathbf{N}$ & $\%$ & $\mathbf{N}$ & $\%$ & & \\
\hline AA & 16 & 35.56 & 29 & 64.44 & - & 1.00 (Reference) \\
AG & 37 & 26.81 & 101 & 73.19 & 0.2631 & $0.664(0.324-1.360)$ \\
GG & 35 & 39.33 & 54 & 60.67 & 0.6713 & $1.175(0.558-2.472)$ \\
AG+GG & 72 & 31.72 & 155 & 68.28 & 0.6155 & $0.842(0.430-1.648)$
\end{tabular}

Four late complications of T2DM in anamnesis were taken into consideration: retinopathy, nephropathy, peripheral neuropathy and macroangiopathy. The conducted analysis provided results as presented below.

Retinopathy occurred in 92 patients (33,8\%). Regarding all of the patients, there were more without the retinopathy among $A A$ and $A G$ genotype. The above indicates more people with genotype $G G$ developing this T2DM complication (Tab. 3).

Nephropathy was present in 26 cases $(9,6 \%)$. Out of the patients who did not suffer from nephropathy $51 \%$ stated AG genotype. The greatest difference can be seen in the GG genotype: $46 \%$ among a group of occurred by retinopathy to only $31 \%$ among patients without this complication (Tab. 4).

Considering the next studied complication, 88 patients of the T2DM group had neuropathy, which is $32,3 \%$ of all. With a slight difference of occurrence in AA genotype, the other two presents as follows: more non-neuropathy patients in comparison to ones with neuropathy in AG genotype and consequently, less patient without this complication than those with neuropathy in GG genotype (Tab. 5).

Macroangiopathy showed most often occurrence. $37,9 \%$ of the studied patients (103) had already been diagnosed with the disease while genotype determining. In this case, the group with $A A$ and $G G$ genotype have not significantly more patients with the complication (Tab. 6).

$24(8,8 \%)$ of patients suffered from macroangiopathy and neuropathy altogether. Most of them presented GG genotype (45,8\%), second AG $(37,5 \%)$ and $16,7 \%$ AA genotype.

Only $6(2,2 \%)$ individuals presented all of the mentioned complications.

We may note among all of the studied complications is that in the AG genotype group there are always more patients without the disease than with one. Exactly opposite we may note in GG genotype in all of the studied cases.

No statistically significant differences in genotype distribution between groups in all researched areas have been observed. 
Table 6. Distribution of genotypes of visfatin's SNP in rs4730153 in reference to macroangiopathy occurrence

\begin{tabular}{lcccccc}
\hline Genotypes & \multicolumn{2}{c}{$\begin{array}{c}\text { With } \\
\text { macroangiopathy }\end{array}$} & \multicolumn{2}{c}{$\begin{array}{c}\text { Without } \\
\text { macroangiopathy }\end{array}$} & p-value & OR (95\% CI) \\
\cline { 2 - 5 } & $\mathbf{N}$ & $\%$ & $\mathbf{N}$ & $\%$ & & \\
\hline $\mathrm{AA}$ & 19 & 42.22 & 26 & 57.78 & - & 1.00 (Reference) \\
$\mathrm{AG}$ & 49 & 35.51 & 89 & 64.49 & 0.4190 & $0.753(0.379-1.497)$ \\
$\mathrm{GG}$ & 35 & 39.33 & 54 & 60.67 & 0.7469 & $0.887(0.428-1.838)$ \\
$\mathrm{AG}+\mathrm{GG}$ & 84 & 37.00 & 143 & 63.00 & 0.5103 & $0.804(0.420-1.540)$ \\
\hline
\end{tabular}

\section{Discussion}

The study aimed to investigate the correlation between different variants of the visfatin gene in rs 4730153 and the occurrence of late complications of T2DM in the population of Southern Poland. The authors wanted to assess whether the studied SNP of the visfatin gene is associated with an increased risk of developing late complications of diabetes.

T2DM is a growing problem nowadays; in 2013 there were 3 million patients diagnosed with diabetes in Poland [11]. What is more, $30 \%$ of patients diagnosed with myocardial infarction have also diabetes; one in seven patients with newly diagnosed diabetes will develop acute coronary syndrome in the next 10 years, $60 \%$ of patients with a duration of diabetes of more than 15 years have retinopathy and $15 \%$ - nephropathy [12].

As has been mentioned, the level of proinflammatory visfatin is elevated in patients with diabetes mellitus. Many studies indicate that visfatin may lead to vascular disorders in different mechanisms: visfatin ability to induce MMP- 9 and nuclear factor $-\kappa$ B which are involved in the instability of atherosclerotic plaque.

Moreover, visfatin may be engaged in endothelial dysfunction [13]. It has been shown that in diabetic macroangiopathy the level of serum visfatin is significantly lower in comparison to patients with non-complicated diabetes. Additionally, the serum level of visfatin can be negatively correlated with the lipid profile [14].

It was described that visfatin as an adipokine with proangiogenic features may take some role in the pathogenesis of diabetic retinopathy. In patients with diabetic retinopathy, the concentration of visfatin in serum and vitreous is elevated and correlated with the severity of the disease [15].

It has been reported that visfatin may stimulate the expression of endothelial nitric oxide in renal cells in patients with diabetic nephropathy. This observation seems to be confirmed by the fact that patients with diabetic nephropathy have an increased level of visfatin in serum [16] especially in the IV stage of disease in comparison to stage III [17]. The level of visfatin in serum can predict the severity of diabetic nephropathy.
There are only several pieces of research that investigated the role of visfatin polymorphism in rs4730153. One of them indicates that there is no association between T2DM and studied SNP, however, the ratio visceral/subcutaneous visfatin expression is correlated with visfatin polymorphism in rs4730153 [18].

Other studies note the role of rs4730153 in the pathogenesis of obesity; it was also described that plasma visfatin level is increased in patients with obesity. One study indicates that genotype AA in rs4730153 of visfatin gene may decrease the risk of cardiovascular disorders both in patients with normal body weight and with obesity. Variant AA of the rs4730153 is related to fasting blood glucose, fasting blood insulin and HOMA-IR (homeostasis model assessment-insulin resistance) [19].

Other research was carried out on Chinese obese children taking part in the aerobic exercise training program. It has observed statistically significant differences in the level of triacylglycerols (TG) and HOMA- value before and after the exercise programme according to genotype in rs4730153. Genotype GG seems to reduce TG level and increase sensitivity to insulin-induced by exercises [20].

There are contradictory data on the association between rs4730153 and BMI; some studies suggest that there is no association between rs4730153 and BMI [18], but on the other hand another one shows a borderline significant correlation between rs4730153 and decreased BMI [21].

The presented study was carried out since there are no other available studies about the role of visfatin polymorphism in rs4730153 in long term complications of diabetes mellitus. This study is the first one that investigated the association between visfatin polymorphism and late complications of diabetes. The authors had not observed any statistically significant association between different variants of the visfatin gene in rs 4720153 and the occurrence of late complications of diabetes. However, the duration of diseases and intensity of glycemic control influence strongly the development of diabetes complications [22]. Also, the disproportion in the number of patients 
with/without specific complications could have affected the results.

This study had some limitations: the concentration of visfatin in serum was not measured; the expression of visfatin by measuring visfatin mRNA was also not examined, so it could not have been assessed how studied SNP influences visfatin expression. Further studies with a complete assessment of visfatin expression not only in serum but also in organs involved in diabetic complications are necessary to assess the role of visfatin polymorphism in rs4730153 in the development of long term complications of diabetes mellitus.

In comparison to other visfatin's SNPs, rs4720153 is yet to be widely researched.

\section{Conclusions}

Collected data showed that SNP in rs4730153 of visfatin using $\chi 2$ test does not show a statistically significant correlation in any of the late complications of T2DM. All of the complications were present more often in heterozygote patients (AG) which may result from the largest group of patient with the genotype. Most tested patients were obese and suffered already from hypertension and dyslipidaemia.

Because of a quite small group and not much of any other research on this polymorphism, further study is needed to know the role of SNP of visfatin in T2DM late complications.

\section{Acknowledgements: The authors would like to thank Władysław Grzeszczak and the Department of Internal Medicine, Diabetology and Nephrology in Zabrze for the idea and for helping financially with the research. The authors wish to show their appreciation to Nikola Szweda who supported them in every part of the research. The authors thank for support in laboratory: Wanda Trautsolt and Sylwia Górczyńska-Kosiorz.}

\section{List of abbreviations:}

$$
\begin{aligned}
& \text { T2DM - Type } 2 \text { Diabetes Mellitus } \\
& \text { WHO - World Health Organization } \\
& \text { SNP - single nucleotide polymorphism } \\
& \text { BMI - Body Mass Index } \\
& \text { WHR - Waist to hip ratio } \\
& \text { TG - triacylglycerols }
\end{aligned}
$$

HOMA-IR - homeostasis model assessment-insulin resistance

$$
\begin{aligned}
& \text { Statement of competing interests: The authors } \\
& \text { report no competing interests. }
\end{aligned}
$$

\section{References}

1. Kharroubi AT, Darwish HM. Diabetes mellitus: The epidemic of the century. World J Diabetes. 2015; 6(6): 850-867, doi: 10.4239/wjd. v6.66.850, indexed in Pubmed: 26131326.

2. International Diabetes Federation. IDF Diabetes Atlas, 8th ed. Brussels.

3. American Diabetes Association. 11. Microvascular Complications and Foot Care: . Diabetes Care. 2019; 42(Suppl 1): S124-S138, doi: 10.2337/dc19-S011, indexed in Pubmed: 30559237.

4. Sorrentino FS, Matteini S, Bonifazzi C, et al. Diabetic retinopathy and endothelin system: microangiopathy versus endothelial dysfunction. Eye (Lond). 2018; 32(7): 1157-1163, doi: 10.1038/s41433-018-0032-4, indexed in Pubmed: 29520046

5. Persson F, Rossing P. Diagnosis of diabetic kidney disease: state of the art and future perspective. Kidney Int Suppl (2011). 2018; 8(1): 2-7, doi: 10.1016/j.kisu.2017.10.003, indexed in Pubmed: 30675433.

6. Ahlqvist E, van Zuydam NR, Groop LC, et al. The genetics of diabetic complications. Nat Rev Nephrol. 2015; 11(5): 277-287, doi: 10.1038/nrneph.2015.37, indexed in Pubmed: 25825086

7. Wang $Y$, Yuan Ye, Jiang $H$. Serum and vitreous levels of visfatin in patients with diabetic retinopathy. Med Sci Monit. 2014; 20: 2729-2732, doi: 10.12659/MSM.891292, indexed in Pubmed: 25524991.

8. Mahmood N, Junejo AM, Jamal Q, et al. Association of visfatin with chronic kidney disease in a cohort of patients with and without diabetes. J Pak Med Assoc. 2010; 60(11): 922-6.

9. Kang YS, Cha DR. The role of visfatin in diabetic nephropathy. Chonnam Med J. 2011; 47(3): 139-143.

10. Mageswari $R$, Sridhar MG, Nandeesha $H$, et al. Irisin and Visfatin Predicts Severity of Diabetic Nephropathy. Indian J Clin Biochem. 2019; 34(3): 342-346, doi: 10.1007/s12291-018-0749-7, indexed in Pubmed: 31391726

11. Cukrzyca. Ukryta pandemia. Sytuacja w Polsce. : Edycja.

12. Kalbarczyk WP, Okopień B. Cukrzyca: gdzie jesteśmy? dokąd zmierzamy?: Raport Instytutu Ochrony Zdrowia. Warszawa: Instytut Ochrony Zdrowia. ; 2018.

13. Liang $\mathrm{W}, \mathrm{Ye} \mathrm{DD}$. The potential of adipokines as biomarkers and therapeutic agents for vascular complications in type 2 diabetes mellitus. Cytokine Growth Factor Rev. 2019; 48: 32-39, doi: 10.1016/j. cytogfr.2019.06.002, indexed in Pubmed: 31229411.

14. Alghasham AA, Barakat YA. Serum visfatin and its relation to insulin resistance and inflammation in type 2 diabetic patients with and without macroangiopathy. Saudi Med J. 2008; 29(2): 185-192, indexed in Pubmed: 18246224.

15. Wang $Y$, Yuan Ye, Jiang $H$. Serum and vitreous levels of visfatin in patients with diabetic retinopathy. Med Sci Monit. 2014; 20: 2729-2732, doi: 10.12659/MSM.891292, indexed in Pubmed: 25524991.

16. Akbarian N, Zarghami N, Mota A, et al. Correlation Between Circulating Visfatin and Nitric Oxide Metabolites Levels in Patients With Diabetic Nephropathy. Iran J Kidney Dis. 2018; 12(3): 163-168, indexed in Pubmed: 29891746

17. Mageswari R, Sridhar MG, Nandeesha $H$, et al. Irisin and Visfatin Predicts Severity of Diabetic Nephropathy. Indian J Clin Biochem. 2019; 34(3): 342-346, doi: 10.1007/s12291-018-0749-7, indexed in Pubmed: 31391726.

18. Böttcher $Y$, Teupser D, Enigk B, et al. Genetic variation in the visfatin gene (PBEF1) and its relation to glucose metabolism and fat-depot-specific messenger ribonucleic acid expression in humans. J Clin Endocrinol Metab. 2006; 91(7): 2725-2731, doi: 10.1210/jc.2006-0149, indexed in Pubmed: 16636125

19. Martínez Larrad MT, Corbatón Anchuelo A, Fernández Pérez C, et al. Segovia Insulin Resistance Study Group (SIRSG). Obesity and Cardiovascular Risk: Variations in Visfatin Gene Can Modify the Obesity Associated Cardiovascular Risk. Results from the Segovia Population Based-Study. Spain. PLoS One. 2016; 11(5): e0153976, doi: 10.1371/journal.pone.0153976, indexed in Pubmed: 27166797.

20. Lai A, Chen W, Helm K. Effects of visfatin gene polymorphism RS4730153 on exercise-induced weight loss of obese children and adolescents of Han Chinese. Int J Biol Sci. 2013; 9(1): 16-21, doi: 10.7150/ijbs.4918, indexed in Pubmed: 23289013.

21. Rong J, Chu M, Xing B, et al. Variations in the gene are associated with body mass index: A population-based study in northern China. Meta Gene. 2015; 6: 65-68, doi: 10.1016/j.mgene.2015.08.004, indexed in Pubmed: 30941280

22. Melmer A, Laimer M. Treatment Goals in Diabetes. Endocr Dev. 2016; 31: 1-27, doi: 10.1159/000439364, indexed in Pubmed: 26824869. 